The Astrophysical Journal, 621:894-901, 2005 March 10

(C) 2005. The American Astronomical Society. All rights reserved. Printed in U.S.A.

\title{
OPTICAL AFTERGLOWS FROM CYLINDRICAL JETS OF SHORT GAMMA-RAY BURSTS
}

\author{
X. Y. WANG, ${ }^{1,2}$ K. S. ChenG, ${ }^{1}$ AND P. H. TAM ${ }^{1}$ \\ Received 2004 September 22; accepted 2004 November 23
}

\begin{abstract}
Observations of extragalactic radio jets and young stellar jets show that the jets are cylindrical; i.e., they maintain a nearly constant cross section on large scales. It has been suggested that the afterglow behaviors of some long-duration gamma-ray bursts (GRBs) are consistent with the cylindrical jet model of GRBs. Here we study the afterglow emission of cylindrical jets from short-duration GRBs. For the usual conical jet geometry, it is argued that, because of the low fluence of short GRBs, the prospects of detecting the optical afterglow $10 \mathrm{hr}$ after the burst are not promising. However, in the present work we find that if the jets are cylindrical, the chance for detecting the optical afterglow will be increased, even if the burst occurs in a low-density $\left(n \sim 10^{-3} \mathrm{~cm}^{-3}\right)$ medium. Since the jets are expected to not be well collimated initially and the time when they change from conical to cylindrical is not exactly known, we discuss two cases for the afterglow of cylindrical jets: the jets becoming cylindrical (1) after the gamma-ray-emitting phase and (2) before the gamma-ray-emitting phase. In both cases, the light-curve behaviors, especially the peak time, are sensitive to the cross section radius of the cylindrical jet. In the former case we find that for viewing angles less than $\sim 0.03 \mathrm{rad}$ relative to the jet axis, typical short GRBs have a late-time $R$-band afterglow with a maximum apparent magnitude of $m_{R} \lesssim 23$, given that the efficiency for producing gamma rays and the shock microphysical parameters of the afterglow are the same in short and long bursts. For the latter case the optical afterglows can always be readily detected with $m_{R}<23$ at $10 \mathrm{hr}$ after the burst. Comparison between model light curves and observational upper limits of the optical afterglow flux of a few short GRBs are also made.
\end{abstract}

Subject headings: gamma rays: bursts — ISM: jets and outflows — radiation mechanisms: nonthermal

\section{INTRODUCTION}

It has been recognized for two decades that the duration distribution of gamma-ray bursts (GRBs) appears to be bimodal, namely, short-duration $(\sim 0.2 \mathrm{~s})$, comprising about $25 \%$ of the total, and long-duration ( $20 \mathrm{~s})$, comprising about $75 \%$ (Mazets et al. 1981; Kouveliotou et al. 1993). The energy spectra of these two classes of bursts are also different: the short bursts tend to have harder spectra, while the long bursts tend to have softer spectra (Kouveliotou et al. 1993; Dezalay et al. 1996). The detection of afterglows at long wavelengths for long-duration GRBs has established their cosmological origin (van Paradijs et al. 1997). The association of long GRBs with star-forming regions (Bloom et al. 2002), and particularly the unambiguous association of GRB 030329 with SN 2003dh (Stanek et al. 2003; Hjorth et al. 2003), supports massive stars as the progenitors of long bursts. However, this remarkable progress is restricted to the long-duration class of GRBs. No counterparts at longer wavelengths have yet been found for the short-duration class bursts, in spite of intense efforts to detect the optical, infrared, and radio counterparts of several short bursts (e.g., Kehoe et al. 2001; Hurley et al. 2002; Castro-Tirado et al. 2002; Gorosabel et al. 2002; Klotz et al. 2003).

The existence of two distinct populations of GRBs might very well be an indication of the presence of two distinct types of progenitors. It is widely accepted that the long bursts are the result of the collapse of massive stars (Woosley 1993; Paczyński 1998; MacFadyen \& Woosley 1999). For the short bursts, the currently favored model involves neutron star-neutron star (NS-NS) mergers or neutron star-black hole (NS-BH) mergers, as simulations of mergers of two compact objects have shown that the

\footnotetext{
1 Department of Physics, University of Hong Kong, Hong Kong, China.

2 Department of Astronomy, Nanjing University, Nanjing 210093, China.
}

duration of the neutrino-driven wind possibly producing the GRB is less than a second (e.g., Ruffert \& Janka 1999; Rosswog et al. 2003). According to this picture, we can also expect that the two classes of bursts might occur in different environments. Massive stars have short lifetimes, and therefore long GRBs are expected to occur in dense and dusty environments, while short bursts involving the merging of NS-NS or NS-BH binaries may occur in the low-density halo of the host galaxy, given the few hundred kilometers per second acquired by the NS at birth and a coalescence time of about $100 \mathrm{Myr}^{3}$

On these grounds, searching for the afterglows of short GRBs is crucial to understanding their nature, as a positive detection can give much information, such as the distance, by determining the redshift, and the burst position relative to the host galaxy. A comprehensive search for the afterglows of short GRBs could be done after the launch of the Swift mission in the near future, as it has the ability to detect and locate a large number of short GRBs quickly enough that the narrow field of view instruments can follow up. However, Panaitescu et al. (2001) and Rosswog et al. (2003) recently argued that the prospect for detecting optical and radio afterglows of short GRBs $10 \mathrm{hr}$ after the burst is not promising. They found that the afterglow emission is $10-100$ times dimmer than that of a long burst at the same epoch, assuming that the efficiency for producing gamma rays and the shock microphysical parameters are the same in short and long GRBs. Panaitescu et al. (2001) predicted $m_{R} \gtrsim$ $2310 \mathrm{hr}$ after the burst, and even dimmer if short GRBs occur in a low-density medium, as favored by the merger scenario of compact objects.

\footnotetext{
${ }^{3}$ These numbers are, however, uncertain (Perna \& Belczynski 2002), and a new class of short-lived NS-NS binaries has been suggested by Belczynski \& Kalogera (2001).
} 
In the calculations of Panaitescu et al. (2001) and Rosswog et al. (2003), a conical geometry for the jets has been assumed as usual. However, we should note that the structure of GRB jets has not been resolved and might not necessarily be conical. Observations of extragalactic radio jets (Bridle \& Perley 1984) and young stellar jets (e.g., Burrows et al. 1996; Ray et al. 1996) indicate that their outflows show a clear cylindrical morphology, i.e., they maintain nearly constant cross sections on large scales, rather than nearly constant opening angles. Theoretically, the jets may be collimated, for example, by the thermal pressure of the external medium or by the toroidal magnetic field in the jets (Bridle et al. 1986). Numerical simulations of the ultrarelativistic magnetohydrodynamic outflow from the BH-disk system of a GRB central engine also show that the jets asymptotically tend to be cylindrical (Vlahakis \& Königl 2003a, 2003b; Fendt \& Ouyed 2004). Interestingly, Cheng et al. (2001) have suggested that the afterglow behavior of some long-duration GRBs is consistent with the cylindrical jet model (for details, see also Huang et al. 2002).

Therefore, afterglow emission from cylindrical jets from short GRBs is worth exploring. Since the jets are expected to not be well collimated initially and it is not known whether the jets have become cylindrical at the gamma-ray-emitting phase, we consider two cases: (1) the gamma-ray-emitting jets are still conical, and (2) the gamma-ray-emitting jets have become cylindrical. The two cases have quite different beaming angles for the emission of prompt gamma rays. For case 1 the effective gamma-ray emission angle is just the opening angle of the jets, $\theta_{0}$, while for case 2 the beaming angle is $1 / \gamma_{0}$, where $\gamma_{0}$ is the initial Lorentz factor of the jets. Moreover, for case 1, as the jet has become cylindrical during the afterglow phase, an observer within the gamma-ray-emitting cone may not be on the axis of the cylindrical jet; that is, although the observer may see the usual burst of gamma rays, he is very likely to see an off-axis afterglow.

In $\S 2$ we present the model assumption and the parameter values used in the calculation of the optical afterglow emission. In $\S 3$ we first give an analytic estimate of the afterglow flux for both cases in order to compare with the results predicted for a conical jet, and then we present the numerical results and compare them with the observations of some short GRBs in $\S 4$. Finally, we give our conclusions and discussion.

\section{MODEL ASSUMPTIONS AND PARAMETER VALUES}

As in the standard theory of GRBs, the afterglow emission of a cylindrical jet is produced by the shock-accelerated electrons in the matter swept up by the forward blast wave, which is formed when the ultrarelativistic GRB ejecta expands into the surrounding medium. The dynamics of the cylindrical jet can be described by the following equation, which is found to be correct for both adiabatic and highly radiative shocks and in both relativistic and Newtonian phases (Huang et al. 1999; Cheng et al. 2001):

$$
\frac{d \gamma}{d m}=-\frac{\gamma^{2}-1}{M_{\mathrm{ej}}+\epsilon m+2(1-\epsilon) \gamma m},
$$

where $\gamma$ is the Lorentz factor of the jet material, $m$ is the mass of the swept-up interstellar medium, $M_{\mathrm{ej}}$ is the mass of the original ejecta, and $\epsilon$ is the radiative efficiency. In this paper we assume that the dynamic evolution of the shock is adiabatic; i.e., $\epsilon=0$. This assumption is correct, since in our model the energy density of the electrons accelerated by the shock is taken to be a fraction $\epsilon_{e} \ll 1$ of the total energy density of the postshock fluid. The evolution of the radius $(R)$ and the swept-up mass are respectively described by (Cheng et al. 2001)

$$
\begin{gathered}
\frac{d R}{d t}=\frac{\beta c}{1-\beta \cos \Theta}, \\
\frac{d m}{d R}=\pi a^{2} n m_{p},
\end{gathered}
$$

where $t$ is the observer time at a viewing angle $\Theta$ from the jet axis, $n$ is the number density of the surrounding medium, $\beta=$ $\left(\gamma^{2}-1\right)^{1 / 2} / \gamma$ is the velocity of the jet in units of the speed of light $c, m_{p}$ is the proton mass, and $a$ is the radius of the cross section of the cylindrical jet. We assume the jets are well collimated and strictly maintain a constant cross section; that is, no sideways expansion effect is taken into account here.

The afterglow emission depends on both the dynamics of the jet and the radiation process. Explicitly, it depends on the following quantities: the kinetic energy $E_{0}$, the initial Lorentz factor $\gamma_{0}$, the lateral radius $a$, the surrounding medium density $n$, and the parameters related to the shock process, such as the equipartition factors of the magnetic field energy density $\left(\epsilon_{B}\right)$ and electron energy density $\left(\epsilon_{e}\right)$ of the shock and the energy distribution index $p$ of the shocked electrons (i.e., $d n_{e} / d \gamma_{e} \propto \gamma_{e}^{-p}$ ). GRB observations show that short bursts have, on average, gamma-ray fluences about 20 times lower than those of long bursts. Like Panaitescu et al. (2001), we assume that the efficiency of converting the outflow's kinetic energy into gamma photons is the same as for long bursts and adopt an isotropic kinetic energy; $E_{\text {iso }}=5 \times 10^{51}$ ergs for a typical short burst at redshift $z=1$ in our calculation. The microphysical parameters of the relativistic shocks are also chosen to be like those typical of long bursts, i.e., $\epsilon_{e}=0.1, \epsilon_{B}=0.01$, and $p=2.2$ (e.g., Wijers \& Galama 1999; Granot et al. 1999; Wang et al. 2000; Panaitescu \& Kumar 2001, 2002). We adopt the hypothesis that short GRBs originate from the merging of binary compact objects in the low-density outskirts of the host galaxy, and so in our calculation the surrounding medium is chosen to be a constant-density medium with $n=10^{-3} \mathrm{~cm}^{-3}$. Throughout the paper the luminosity distance is chosen to be $d_{L}=10^{28} \mathrm{~cm}$. Real magnetically driven jets from a central engine may be quasi-universal and structured, with a Gaussian-like or similar structure (Zhang \& Mészáros 2002; Rossi et al. 2002; Kumar \& Granot 2003; Zhang et al. 2004), but as a first approximation and for comparison with the result of Panaitescu et al. (2001), we assume a uniform jet in our calculation.

Little is known about the size of the lateral radius $a$ of the cylindrical jets. The numerical simulations of the outflow from the GRB central engine by Vlahakis \& Königl (2003a, 2003b) and Fendt \& Ouyed (2004) only extend to the end of the acceleration phase, well before the time when the afterglow phase begins. There are two ways to give a naive estimate of the value of $a$. One is to make a simple analogy with extragalactic radio jets, for which observations show that the ratio between the cross section radius and the jet length is between $\lesssim 0.01$ and $\sim 0.1$ (Bridle \& Perley 1984). Assuming that the ratio between $a$ and the deceleration radius of the jet $\left(a / r_{\mathrm{dec}}\right)$ has the same magnitude in the short-GRB case, $a$ is about $10^{15}-10^{16} \mathrm{~cm}$ during the afterglow phase for a jet propagating in a low-density medium. Another way is to assume the jets are collimated by the pressure of the external medium. Equilibrium between the internal magnetic field pressure and that of the external medium, 
as suggested in Beskin (1997) for extragalactic radio jets and young stellar jets, leads to

$$
\frac{B_{\mathrm{in}}^{2}}{8 \pi}\left(\frac{a}{R_{0}}\right)^{-4} \sim n k T \sim \frac{B_{\mathrm{ext}}^{2}}{8 \pi},
$$

where $B_{\text {in }}$ and $R_{0}$ are respectively the radius and the magnetic field of the central compact object, $n k T$ is the gas pressure of the external medium, and $B_{\text {ext }}$ is the external galactic magnetic field. Therefore, $a \sim R_{0}\left(B_{\text {in }} / B_{\text {ext }}\right)^{1 / 2}$ (Beskin 1997; Lobanov et al. 2001). For the BH-disk system produced by the NS-NS merger, $B_{\text {in }} \sim 10^{12}-10^{13} \mathrm{G}, B_{\text {ext }} \sim 10^{-6} \mathrm{G}$, and $R_{0} \sim 10^{6} \mathrm{~cm}$, we get $a \sim 10^{15}-10^{16} \mathrm{~cm}$. In the numerical study below, we choose three representative values: $a=10^{14}, 10^{15}$, and $10^{16} \mathrm{~cm}$.

\section{ANALYTIC ESTIMATE OF THE AFTERGLOW EMISSION}

It is naturally believed that the jets are initially conical and later become cylindrical by some effective collimation mechanism. Although we consider the jets to be cylindrical on large scales, i.e., during the afterglow phase, we know little about the jet structure during the early gamma-ray-emitting phase. In other words, the jets in the gamma-ray-emitting phase could be cylindrical or could still be conical before becoming cylindrical in the afterglow phase. Because these two cases have different implications for the observed afterglow fluxes, we discuss them separately. We define the case in which the gamma-ray-emitting jets are still conical as case 1 and the case in which the gammaray-emitting jets have already become cylindrical as case 2 . For case 1 the gamma-ray emission angle is just the opening angle of the jets, $\theta_{0}$; observers with viewing angles within $\theta_{0}$ receive the same gamma-ray fluence as observers on the jet axis. But when the jets become cylindrical during the afterglow phase, the beaming angle becomes $1 / \gamma$, where $\gamma$ is the Lorentz factor of the jets. Since during the afterglow phase $1 / \gamma$ is likely much smaller than $\theta_{0}$, observers outside the cone of $1 / \gamma$ (but still within $\theta_{0}$ ) will see a much dimmer afterglow flux than those on the jet axis, although both observers would receive about the same gamma-ray fluence. Moreover, the probability for the observers to be outside the beaming angle $1 / \gamma$ is much larger than that to be within it; in other words, observers are more likely to observe an off-axis afterglow from the cylindrical jet. Case 2 is much simpler, because the jets during the gamma-ray-emitting phase and the afterglow phase are both cylindrical. An observer within the beaming angle of the gamma rays $\left(1 / \gamma_{0}\right)$ is always within the emission angle of the afterglow $(1 / \gamma)$ because the Lorentz factor is decreasing with time $\left(\gamma_{0} \geq \gamma\right)$.

\subsection{Case 1}

We first estimate the optical afterglow flux received by an observer whose line of sight is along the jet axis, i.e., $\Theta=0$. Following the calculation of Panaitescu et al. (2001), we assume an average $25 \mathrm{keV}-1 \mathrm{MeV}$ fluence of $\sim 0.5 \times 10^{-6} \mathrm{ergs} \mathrm{cm}^{-2}$ for a typical short burst, so the isotropic kinetic energy of the burst at the gamma-ray-emitting phase is $E_{\text {iso }}=5 \times 10^{51} d_{L, 28}^{2} \mathrm{ergs}$, and the real kinetic energy of the jet is therefore

$$
\begin{aligned}
E & =E_{\text {iso }}\left(1-\cos \theta_{0}\right) \simeq E_{\text {iso }} \theta_{0}^{2} / 2 \\
& =6 \times 10^{48}\left(\theta_{0} / 0.05\right)^{-2} d_{L, 28}^{2} \text { ergs. }
\end{aligned}
$$

This energy is consistent with the neutrino annihilation energy deposited into the outflow in the NS-NS merger simulations of Ruffert \& Janka (1999) and Rosswog et al. (2003). The amount of energy extracted by magnetic mechanisms from the
BH-disk system is more uncertain (e.g., Rosswog et al. 2003) and can be reconciled with the energy we adopt here as well. We have chosen the opening angle $\theta_{0}$ of the jets of short GRBs to be the average value for long GRBs, $\theta_{0} \simeq 0.05 \mathrm{rad}$, obtained by Frail et al. (2001) from the jet break time of the afterglow. The value $\theta_{0} \simeq 0.05 \mathrm{rad}$ is also consistent with that derived from the comparison of the observationally inferred local coalescence rate of NS-NS binaries, which was recently found to be rather large (180 -144 $\mathrm{Myr}^{-1}$ galaxy $^{-1}$; Kalogera et al. 2004), and the estimate obtained by theoretical population synthesis (Ando 2004). For the initial Lorentz factor $\gamma_{0}$ of GRBs, we chose a representative value of 300 here.

A forward-moving blast wave is produced by the interaction of the jet from the central engine of the GRB with the surrounding medium. Synchrotron radiation from the shock-accelerated electrons in the medium swept up by the blast wave gives rise to the GRB afterglow (Waxman 1997a, 1997b; Wijers et al. 1997; Mészáros \& Rees 1997; Vietri 1997). The cylindrical jet will be decelerated by the swept-up surrounding medium at the radius

$$
\begin{aligned}
R_{\mathrm{dec}} & =\frac{E / \gamma_{0} c^{2}}{\pi a^{2} n m_{p}} \\
& =1.4 \times 10^{19}\left(\frac{\gamma_{0}}{300}\right)^{-2}\left(\frac{E}{6 \times 10^{48} \mathrm{ergs}}\right) a_{15}^{-2} n_{-3}^{-1} d_{L, 28}^{2} \mathrm{~cm}
\end{aligned}
$$

and at the time

$t_{\mathrm{dec}}=\frac{R_{\mathrm{dec}}}{2 \gamma_{0}^{2} c}=2.4 \times 10^{3}\left(\frac{\gamma_{0}}{300}\right)^{-4}\left(\frac{E}{6 \times 10^{48} \mathrm{ergs}}\right) a_{15}^{-2} n_{-3}^{-1} d_{L, 28}^{2} \mathrm{~s}$,

where the usual notation $C_{n}=C / 10^{n}$ in cgs units is used here (and also throughout this work). We discuss below the afterglow evolution for $t<t_{\mathrm{dec}}$ and $t>t_{\mathrm{dec}}$, respectively.

Before the deceleration $\left(t<t_{\mathrm{dec}}\right)$, the Lorentz factor of the jet can be regarded as constant, with $\gamma=\gamma_{0}$, so the radius is $R=$ $2 \gamma_{0}^{2} c t$. As usual, we assume that the energy density acquired by the magnetic field and the shocked electrons are respectively the fractions $\epsilon_{B}$ and $\epsilon_{e}$ of the total thermal energy in the shocked medium, which means that the magnetic field in the comoving frame is

$$
B^{\prime}=\sqrt{32 \pi \epsilon_{B} \gamma_{0} n m_{p} c^{2}}=0.37 \epsilon_{B,-2}^{1 / 2}\left(\frac{\gamma_{0}}{300}\right) n_{-3}^{1 / 2} \mathrm{G},
$$

and the minimum Lorentz factor of the random motion of electrons in the comoving frame is

$$
\gamma_{m}=\epsilon_{e} \frac{p-2}{p-1} \frac{m_{p}}{m_{e}} \gamma=9 \times 10^{3} \epsilon_{e,-1}\left(\frac{\gamma_{0}}{300}\right)
$$

for $p=2$.2. Sari et al. (1998) have defined a critical Lorentz factor $\gamma_{c}=6 \pi m_{e} c /\left(\sigma_{\mathrm{T}} \gamma B^{\prime 2} t\right)$ above which synchrotron radiation is significant. The distribution of the electrons with $\gamma_{e}>\gamma_{c}$ becomes steeper with $d n_{e} / d \gamma_{e} \propto \gamma_{e}^{-(p+1)}$. Then we can obtain the characteristic frequencies corresponding to $\gamma_{m}$ and $\gamma_{c}$, respectively, i.e.,

$$
\begin{aligned}
& \nu_{m}=2.4 \times 10^{16} \epsilon_{e,-1}^{2} \epsilon_{B,-2}^{1 / 2}\left(\frac{\gamma_{0}}{300}\right)^{4} \mathrm{~Hz}, \\
& \nu_{c}=10^{23}\left(\frac{\gamma_{0}}{300}\right)^{-4} \epsilon_{B,-2}^{-3 / 2} n_{-3}^{-3 / 2} t^{-2} \mathrm{~Hz} .
\end{aligned}
$$


The peak flux of the synchrotron radiation from a cylindrical jet is given by

$$
\begin{aligned}
F_{\nu_{m}} & =\frac{N_{e} \gamma P_{\nu_{m}}^{\prime}(1+z)}{\Omega d_{L}^{2}} \\
& =1.3 \times 10^{-2}\left(\frac{\gamma_{0}}{300}\right)^{6} a_{15}^{2} n_{-3}^{-3 / 2} \epsilon_{B,-2}^{1 / 2} d_{L, 28}^{-2} t \mathrm{mJy},
\end{aligned}
$$

where $N_{e}=m / m_{p}=\pi a^{2} n R$ is the total number of swept-up electrons, $P_{\nu_{m}}^{\prime}=\sqrt{3} e^{3} B^{\prime} / m_{e} c^{2}$ is the peak spectral power, $\Omega=2 \pi / \gamma_{0}^{2}$ is the solid angle into which the radiation is beamed for a relativistic jet moving with a Lorentz factor of $\gamma_{0}$, and $d_{L}$ is luminosity distance of the burst. During the time $t<t_{\mathrm{dec}}$, the flux of the optical afterglow increases with time as $F_{\text {opt }}=$ $F_{\nu_{m}}\left(\nu / \nu_{m}\right)^{1 / 3} \propto t$. It reaches the peak at $t_{\text {dec }}$. At this time,

$$
\begin{gathered}
F_{\nu_{m}}=30\left(\frac{\gamma_{0}}{300}\right)^{2} n_{-3}^{1 / 2} \epsilon_{B,-2}^{1 / 2}\left(\frac{E}{6 \times 10^{48} \mathrm{ergs}}\right) \mathrm{mJy}, \\
\nu_{m}=2.4 \times 10^{16} \epsilon_{e,-1}^{2} \epsilon_{B,-2}^{1 / 2}\left(\frac{\gamma_{0}}{300}\right)^{4} \mathrm{~Hz}, \\
\nu_{c}=1.8 \times 10^{16}\left(\frac{\gamma_{0}}{300}\right)^{4} \epsilon_{B,-2}^{-3 / 2} n_{-3}^{1 / 2}\left(\frac{E}{6 \times 10^{48} \mathrm{ergs}}\right)^{-2} a_{15}^{4} \mathrm{~Hz} .
\end{gathered}
$$

Here we estimate the optical afterglow flux only for $a=10^{15}$ $\mathrm{cm}$ as a representative case in an analytic way; for $a=10^{14}$ and $10^{16} \mathrm{~cm}$, the numerical results are given in $\S 4$. For $a=10^{15} \mathrm{~cm}$ and typical values for other parameters, we have $\nu_{R}<\nu_{c}<\nu_{m}$, and the optical flux in the $R$ band $\left(\nu_{R}=5 \times 10^{14} \mathrm{~Hz}\right)$ is

$$
\begin{aligned}
F_{R}\left(t_{\mathrm{dec}}\right) & =F_{\nu_{m}}\left(\frac{\nu}{\nu_{c}}\right)^{1 / 3} \\
& =9\left(\frac{\gamma_{0}}{300}\right)^{2 / 3} \epsilon_{B,-2} n_{-3}^{1 / 3}\left(\frac{E}{6 \times 10^{48} \mathrm{ergs}}\right)^{5 / 3} d_{L, 28}^{4 / 3} \mathrm{mJy},
\end{aligned}
$$

or the apparent magnitude of the $R$ band is $m_{R} \simeq 14$.

After the deceleration time $\left(t>t_{\mathrm{dec}}\right)$, the cylindrical jet decelerates significantly. During this phase, the dynamics is different: $\gamma \propto t^{-1 / 4}$, and $R \propto t^{1 / 2}$ (Cheng et al. 2001). In addition, we get $B^{\prime} \propto t^{-1 / 4}, \gamma_{m} \propto \gamma \propto t^{-1 / 4}$, and $\gamma_{c} \propto \gamma^{-1} B^{\prime-2} \propto t^{-1 / 4}$. Therefore, $\nu_{m} \propto \gamma \gamma_{m}^{2} B^{\prime} \propto t^{-1}, \nu_{c} \propto \gamma \gamma_{c}^{2} B^{\prime} \propto t^{-1}$, and $F_{\nu_{m}} \propto$ $N_{e} \gamma B^{\prime} / \gamma^{-2} \propto t^{-1 / 2}$ (Cheng et al. 2001). Defining $t_{m}$ as the time when $\nu_{R}=\nu_{c}$, we get

$$
t_{m}=\frac{\nu_{c}\left(t_{\mathrm{dec}}\right)}{\nu_{R}} t_{\mathrm{dec}}=10^{5} \epsilon_{B,-2}^{-3 / 2} n_{-3}^{-1 / 2}\left(\frac{E}{6 \times 10^{48} \mathrm{ergs}}\right)^{-1} d_{L, 28}^{-2} \mathrm{~s} .
$$

When $t_{\text {dec }}<t<t_{m}$, we have $\nu_{R}<\nu_{c}<\nu_{m}$ and $F_{R}=F_{\nu_{m}}(\nu /$ $\left.\nu_{c}\right)^{1 / 3} \propto t^{-1 / 6}$, while when $t>t_{m}$, we have $\nu_{R}>\nu_{m}>\nu_{c}$ and

$$
F_{\nu}=F_{\nu_{m}}\left(\nu_{c} / \nu_{m}\right)^{-(p-1) / 2}\left(\nu / \nu_{c}\right)^{-p / 2} \propto t^{-(p+1) / 2} .
$$

For comparison with the result of Panaitescu et al. (2001), we estimate the expected optical afterglow flux at its peak time after the burst for an off-axis observer at $\Theta=0.03 \mathrm{rad}$. The light curve peaks at the time when $\gamma$ decreases to $\gamma \simeq 1 / \Theta=33$. Since at $t_{\mathrm{dec}}$ the Lorentz factor of the jet is about $(\sqrt{5}-1) / 2 \gamma_{0}=185$, and after that $\gamma \propto t^{-1 / 4}$, the corresponding time for an on-axis observer is $t_{0}=t_{\mathrm{dec}}(185 / 33)^{4}=2.3 \times 10^{6} \mathrm{~s}$. But for the off-axis observer at $\Theta=0.03 \mathrm{rad}$, the peak time is $t_{p}=t_{0} / \kappa \sim 50$ days, where

$$
\kappa \equiv(1-\beta) /(1-\beta \cos \Theta) \simeq 1 /\left(1+\gamma^{2} \Theta^{2}\right)=0.5
$$

(Granot et al. 2002). According to Granot et al. (2002), the received flux at frequency $\nu$ and time $t$ by an observer viewing at an angle $\Theta$ relative to the jet axis is related to that received by an onaxis observer as

$$
F_{\nu}(\Theta, t)=\kappa^{3} F_{\nu / \kappa}(0, \kappa t) .
$$

If $F_{\nu} \propto \nu^{-\beta} t^{-\alpha}(\beta=1.1, \alpha=1.6)$ during the time from $\kappa t$ to $t$, then $F_{\nu}(\Theta, t)=\kappa^{3-\alpha+\beta} F_{\nu}(0, t)$. We estimate that the optical flux at $t_{p}=50$ days for the on-axis observer is

$$
F_{R}=F_{R}\left(t_{\mathrm{dec}}\right)\left(\frac{t_{m}}{t_{\mathrm{dec}}}\right)^{-1 / 6}\left(\frac{50 \text { days }}{t_{m}}\right)^{-(p+1) / 2}=13 \mu \mathrm{Jy} .
$$

By simple algebraic calculation, we find that the magnitude of the observed afterglow flux at $t_{p}=50$ days is $F_{R}=2.3 \mu \mathrm{Jy}$ or $m_{R}=22.8$ for $\Theta=0.03 \mathrm{rad}$. This means that an observer who detected the gamma-ray flash of the short burst has a probability of about $\left(0.03 / \theta_{0}\right)^{2} \simeq 36 \%\left(\theta_{0} / 0.05\right)^{-2}$ of detecting an $m_{R}<22.8$ optical afterglow at the peak time, which is about days to months after the burst. Therefore, we predict that if short GRBs belong to this case, about one-third of short bursts have optical afterglows with a maximum brightness of $m_{R}<23$ for typical parameter values, which could be detected by ground-based optical telescopes.

\subsection{Case 2}

In case 2 , since the jets have become cylindrical after their initial conical geometry at the gamma-ray-emitting phase, an observer seeing a normal burst of gamma rays is within the beaming angle $1 / \gamma_{0}$ relative to the jet axis. The afterglow seen by him is very similar to that for an on-axis observer. The beaming angle of gamma rays depends on the initial Lorentz factor $\gamma_{0}$. Because too low a beaming angle implies a very large intrinsic GRB rate, we here adopt $\gamma_{0}=100$ instead of 300 for this case to avoid too large a rate, but still agree with the estimated NS-NS merger rate within its uncertainty. The isotropic kinetic energy is chosen to be the same as in case $1, E_{\text {iso }}=5 \times$ $10^{51} d_{L, 28}^{2}$ ergs, so that observers could detect the same gammaray fluence given the same efficiency of converting the kinetic energy into gamma-ray photons. The real kinetic energy is thus $E=E_{\text {iso }} /\left(2 \gamma_{0}^{2}\right)=2.5 \times 10^{47} d_{L, 28}^{2}$ ergs, which is consistent with the low end of the outflow energy in simulations of an NS-NS merger (see the corotating run D in Rosswog et al. [2003]).

The deceleration time is

$$
t_{\mathrm{dec}}=8 \times 10^{3}\left(\frac{E}{2.5 \times 10^{47} \mathrm{ergs}}\right) \gamma_{0,2}^{-4} a_{15}^{-2} n_{-3}^{-1} d_{L, 28}^{2} \mathrm{~s} .
$$

At this time, the peak flux and two break frequencies are, respectively,

$$
\begin{gathered}
F_{\nu_{m}}=140\left(\frac{E}{2.5 \times 10^{47} \mathrm{ergs}}\right) \gamma_{0,2}^{2} n_{-3}^{1 / 2} \epsilon_{B,-2}^{1 / 2} \mu \mathrm{Jy}, \\
\nu_{m}=2 \times 10^{14} \epsilon_{e,-1}^{2} \epsilon_{B,-2}^{1 / 2} \gamma_{0,2}^{4} \mathrm{~Hz}, \\
\nu_{c}=1.3 \times 10^{17} \gamma_{0,2}^{4} \epsilon_{B,-2}^{-3 / 2} n_{-3}^{1 / 2}\left(\frac{E}{2.5 \times 10^{47} \mathrm{ergs}}\right)^{-2} d_{L, 28}^{-2} a_{15}^{4} \mathrm{~Hz} .
\end{gathered}
$$




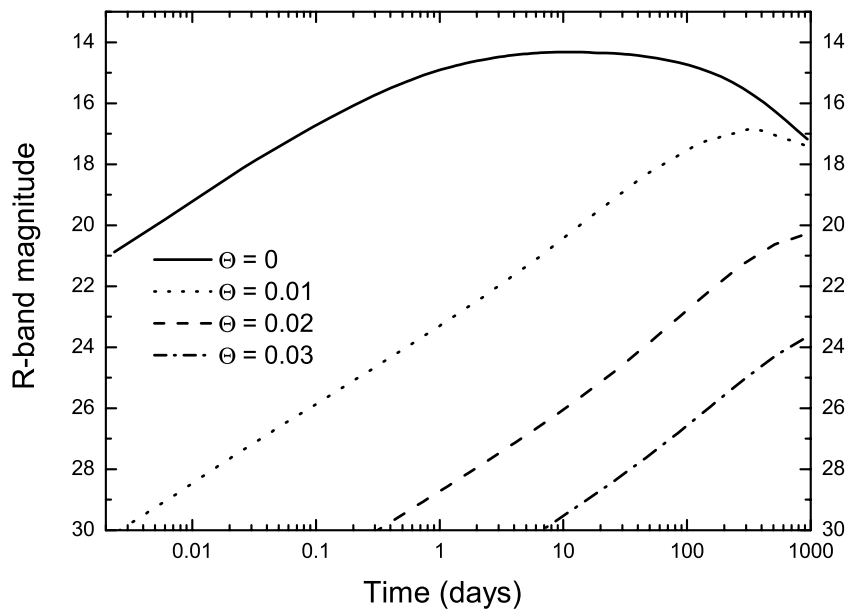

FIG. 1.-Model light curves of a case 1 optical afterglow for $a=10^{14} \mathrm{~cm}$ and for different viewing angles $\Theta$. The shock parameters, surrounding medium density, and luminosity distance are $E=6 \times 10^{48} \mathrm{ergs}, \gamma_{0}=300, \epsilon_{e}=$ $0.1, \epsilon_{B}=0.01, p=2.2, n=10^{-3} \mathrm{~cm}^{-3}$, and $d_{L}=10^{28} \mathrm{~cm}$.

As $\nu_{m}<\nu_{R}<\nu_{c}$, we get the optical flux of the afterglow at $t_{\mathrm{dec}}$,

$$
\begin{aligned}
F_{R} & =F_{\nu_{m}}\left(\frac{\nu}{\nu_{m}}\right)^{-(p-1) / 2} \\
& =80 \gamma_{0,2}^{4.4} n_{-3}^{1 / 2} \epsilon_{e,-1}^{1.2} \epsilon_{B,-2}^{0.8}\left(\frac{E}{2.5 \times 10^{47} \mathrm{ergs}}\right) \mu \mathrm{Jy}
\end{aligned}
$$

for $a=10^{15} \mathrm{~cm}$ and $p=2.2$. Before the deceleration time $\left(t<t_{\mathrm{dec}}\right), F_{R}=F_{\nu_{m}}\left(\nu / \nu_{m}\right)^{1 / 3} \propto t$. After $t_{\mathrm{dec}}$ but before the time when the cooling frequency $\nu_{c}$ crosses the optical band, which is later than $10^{6} \mathrm{~s}$ for typical parameter values, the optical afterglow decays as $F_{R}=F_{\nu_{m}}\left(\nu / \nu_{m}\right)^{-(p-1) / 2} \propto t^{-p / 2}$. Therefore, at $t=10 \mathrm{hr}$ after the burst, the optical afterglow flux is

$$
F_{R}=15\left(\frac{E}{2.5 \times 10^{47} \mathrm{ergs}}\right)^{1.6} n_{-3}^{-0.6} \epsilon_{e,-1}^{1.2} \epsilon_{B,-2}^{0.8} d_{L, 28}^{1.2} \mu \mathrm{Jy}
$$

for $p=2.2$, or a magnitude of $m_{R}=20.8$. After the cooling frequency $\nu_{c}$ crosses the optical band, we have $\nu_{R}>\nu_{c}>\nu_{m}$, and the flux then decays as $F_{\nu} \propto t^{-(p+1) / 2}$.

\section{NUMERICAL CALCULATIONS OF THE AFTERGLOW EMISSION AND COMPARISON WITH OBSERVATIONS}

We use equations (8)-(13) in Cheng et al. (2001) describing the radiation process in our calculation. The observed flux density at a given frequency is given by (Huang et al. 2000)

$$
F_{\nu}=\frac{1}{\gamma^{3}(1-\beta \cos \Theta)^{3}} \frac{1}{4 \pi d_{L}^{2}} P^{\prime}(\gamma(1-\beta \cos \Theta) \nu),
$$

where $P^{\prime}\left(\nu^{\prime}\right)$ is the synchrotron power at $\nu^{\prime}$ in the local frame and is given by

$$
P^{\prime}\left(\nu^{\prime}\right)=\frac{\sqrt{3} e^{3} B^{\prime}}{m_{e} c^{2}} \int_{\gamma_{m}}^{\gamma_{M}}\left(\frac{d N_{e}^{\prime}}{d \gamma_{e}}\right) F\left(\frac{\nu^{\prime}}{\nu_{\mathrm{cr}}^{\prime}}\right) d \gamma_{e}
$$

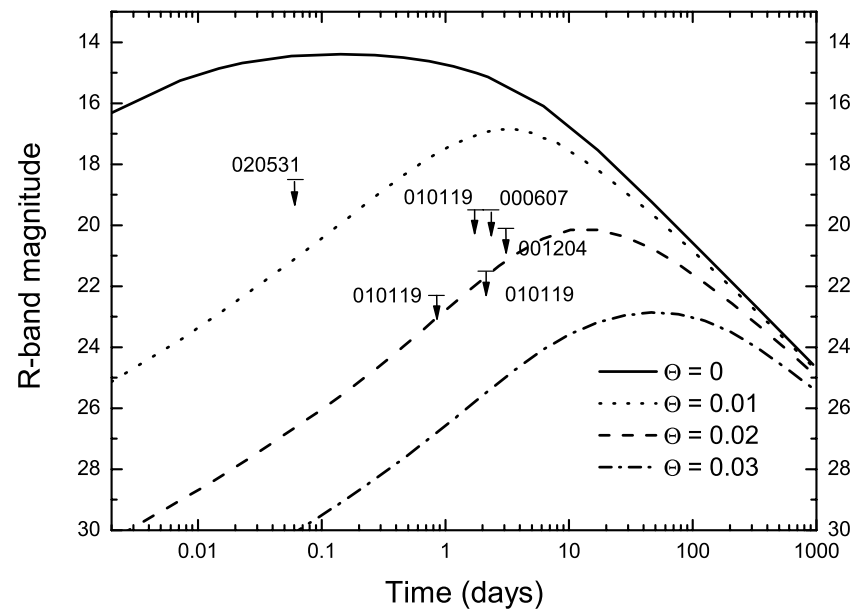

FIG. 2.- Same as Fig. 1, but for $a=10^{15} \mathrm{~cm}$. The most constraining observational upper limits for a few short bursts are also plotted.

where $\gamma_{M}$ is the maximum Lorentz factor of the accelerated electrons, $\nu_{\mathrm{cr}}^{\prime}=3 \gamma_{e}^{2} e B^{\prime} /\left(4 \pi m_{e} c\right)$, and $F(x)=x \int_{x}^{+\infty} K_{5 / 3}\left(x^{\prime}\right) d x^{\prime}$, where $K_{5 / 3}$ is the Bessel function.

\subsection{Case 1}

We calculate the optical afterglow light curves for $a=10^{14}$, $10^{15}$, and $10^{16} \mathrm{~cm}$, shown in Figures $1-3$, respectively. For each case we plot the light curves for different viewing angles $\Theta$ of the observer. For $\Theta=0$, the afterglow increases first and reaches a peak at the deceleration time $t_{\mathrm{dec}}$ of the jet. After that, it begins to decay and shows a steeper decay at later times. This behavior is qualitatively consistent with our analytic study in $\S 3$. As $\Theta$ increases, the flux at a fixed time decreases markedly compared with the on-axis case. Quite unlike that for the conical jet afterglow, this light curve exhibits a longer rising phase to the peak, and after the peak it decays and approaches the onaxis light curve at late times. The rising phase results from the decrease of $\gamma$, which makes the beaming angle increase with time. The peak time, corresponding to the time when $\gamma$ decreases to $\sim 1 / \Theta$, is sensitive to the value of $a$, since for a smaller $a$ the jet decelerates more slowly. It is about 1 day for $\Theta=0.03 \mathrm{rad}$ and $a=10^{16}$, much longer than for the afterglow of a long burst, which is usually shorter than $1 \mathrm{hr}$. The numerical calculations confirm the analytic results that for the same values of

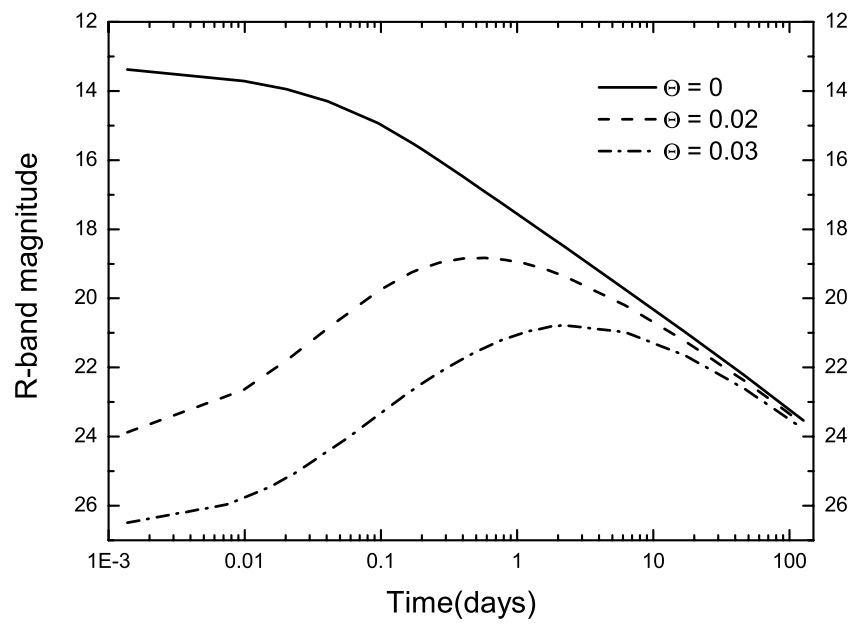

Fig. 3. - Same as Fig. 1, but for $a=10^{16} \mathrm{~cm}$. 


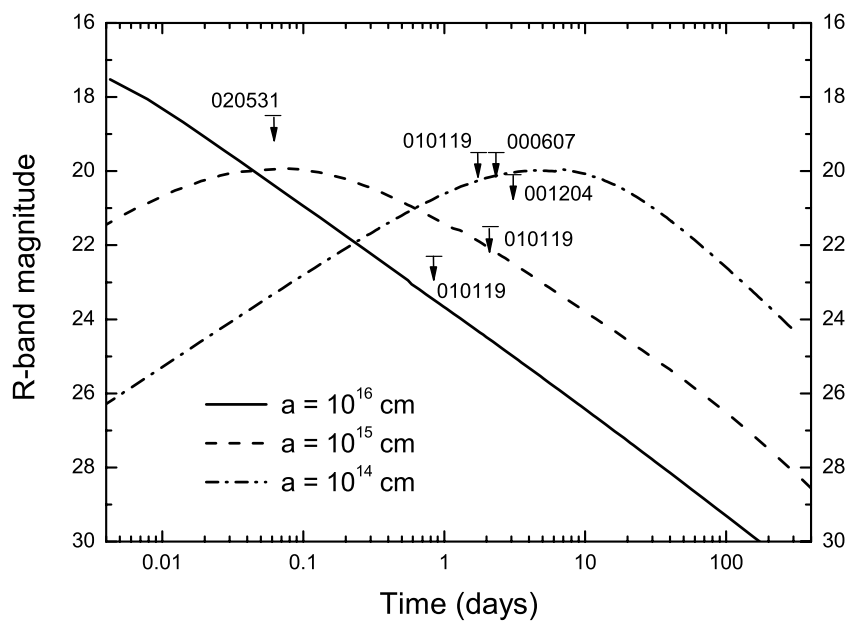

FIG. 4.-Model light curves of a case 2 optical afterglow for different cross section radii $a$ of the cylindrical jets. The shock parameters, surrounding medium density, and luminosity distance are $E=2.5 \times 10^{47} \mathrm{ergs}, \gamma_{0}=100, \epsilon_{e}=$ $0.1, \epsilon_{B}=0.01, p=2.2, n=10^{-3} \mathrm{~cm}^{-3}$, and $d_{L}=10^{28} \mathrm{~cm}$. The observational upper limits for a few short bursts are also plotted.

the isotropic kinetic energy and microphysical parameters, such as $\epsilon_{e}$ and $\epsilon_{B}$ as used in Panaitescu et al. (2001), the optical afterglow from the cylindrical jet is found to be brighter than $m_{R}=23$ at the peak time even for $n=10^{-3} \mathrm{~cm}^{-3}$ when the angle between our line of sight and the jet axis is less than $\Theta=0.03 \mathrm{rad}$. This indicates that about one-third of short GRBs may have optical afterglows with a maximum brightness of $m_{R}<23$ for an average gamma-ray-emitting opening angle $\theta=0.05 \mathrm{rad}$.

\subsection{Case 2}

In this case, the real kinetic energy and the initial Lorentz factor are chosen to be $E=2.5 \times 10^{47}$ ergs and $\gamma_{0}=100$ in order to get the same isotropic kinetic energy $E_{\text {iso }}=5 \times 10^{51}$ ergs. Because for this case observers within the gamma-ray-emitting cone are always within the relativistic beaming angle of the afterglow jets, we only need to consider $\Theta=0$. The calculated light curves are shown in Figure 4 for three representative values of $a$. We can see that for all these values of $a$, the optical flux at $t=10 \mathrm{hr}$ is brighter than $m_{R}=23$. Therefore, the detection of optical afterglows by ground-based telescopes is more promising in this case. The peak time of the light curves, mainly determined by the deceleration time of the jet, is longer for smaller $a$.

\subsection{Comparison with Observations}

It should be noted that for the short/hard GRBs, no optical counterpart has yet been detected in spite of extensive efforts (e.g., Kehoe et al. 2001; Hurley et al. 2002; Castro-Tirado et al. 2002; Gorosabel et al. 2002; Klotz et al. 2003). However, upper limits have been given and should be useful for constraining our model by comparing them with the model light curves. In Figure 2 (as an example of case 1) and Figure 4, we plot the most constraining upper limits of the afterglow emission for short bursts GRB 010119 (Gorosabel et al. 2002; Hurley et al. 2002), GRB 001204 (Hurley et al. 2002), GRB 000607 (Hurley et al. 2002), and GRB 020531 (Klotz et al. 2003). From Figure 2 we can see that the upper limits are consistent with the light curve for $\Theta>0.02 \mathrm{rad}$ in case 1 . We should note that the probability with $\Theta \lesssim 0.02$ rad for observers is only $16 \%\left(\theta_{0} / 0.05\right)^{-2}$. For case 2 (Fig. 4) the upper limits disfavor $a \lesssim 10^{15} \mathrm{~cm}$ but agree with the light curves for $a>10^{15} \mathrm{~cm}$. However, one should be cautious with this conclusion, since in the comparison standard conditions (such as the isotropic energy $E$ and the shock microphysical parameters $\epsilon_{e}$ and $\epsilon_{B}$ ) have been adopted to get the model light curve, while we know that they are likely to vary from burst to burst on the basis of light-curve modeling of longGRB afterglows (e.g., Panaitescu \& Kumar 2001, 2002).

Recently, the High Energy Transient Explorer 2 was triggered by GRB 040924 . This burst had a duration of $T_{50} \sim 1.2 \mathrm{~s}$, and the $7-400 \mathrm{keV}$ fluence was about $4.2 \times 10^{-6} \mathrm{ergs} \mathrm{cm}^{-2}$ (Fenimore et al. 2004; Golenetskii et al. 2004). Unlike traditional short/hard bursts, it has a relatively soft spectrum and can be classified as an X-ray-rich GRB. The optical and infrared afterglows were detected by rapid follow-up observations (e.g., Fox \& Moon 2004; Li et al. 2004) and were well modeled with an isotropic fireball expanding into a low-density medium with $n \sim 0.01 \mathrm{~cm}^{-3}$ (Fan et al. 2004). We find that its afterglow is also consistent with case 2 of our model with $a \sim 10^{16} \mathrm{~cm}$, although the fact that both the duration and fluence of GRB 040924 are between the long and soft classes and short and hard classes of GRBs makes its nature unclear.

\section{DISCUSSION AND CONCLUSIONS}

By analogy with extragalactic radio jets and young stellar jets, a cylindrical jet model for short GRBs is suggested here. However, the physics of how the jet emanating from the central engine becomes cylindrical and acquires the correct cross section radius in the afterglow phase is not well understood. Although numerical simulations of the ultrarelativistic magnetohydrodynamic outflow from the black hole-disk system of a GRB central engine show that the jets tend to become cylindrical asymptotically (Vlahakis \& Königl 2003a, 2003b; Fendt \& Ouyed 2004), the simulations only extend to the end of the acceleration phase, which is well before the time when the afterglow phase begins. At the end of the simulations, the jets have only a very small cross section radius, $a<10^{11} \mathrm{~cm}$. How the cylindrical jet cross section radius widens to more than $10^{14} \mathrm{~cm}$, as required to produce a detectable afterglow, may be related to the propagation through and interaction with the surrounding medium of this magnetohydrodynamic jet and is worth further study, but is, however, beyond the purpose of this paper. Because of this, we have made a simple analogy with extragalactic radio jets when estimating the cross section radius and allow a wide range, $10^{14} \mathrm{~cm} \lesssim a \lesssim 10^{16} \mathrm{~cm}$.

We would like to discuss how to discriminate between cases 1 and 2, and also between cylindrical and conical jets in future observations of the afterglow light curves of short GRBs. The optical afterglow light curve of case 1, when detected for $\Theta>0$, is quite similar to that of case 2 for low values of $a$; that is, they rise to a peak after a long time and then approach a powerlaw decay of $F_{\nu} \propto t^{-(p+1) / 2}$. However, the two peak times have different origins: for case 1 the peak time corresponds to $\gamma\left(t_{\text {peak }}\right) \simeq 1 / \Theta$, while for case 2 the peak time is determined by the deceleration time $t_{\mathrm{dec}}$ of the cylindrical jet. One may be able to discriminate between them if the physical parameters of the afterglow and the medium density can be determined from a multiwavelength spectrum (e.g., Wijers \& Galama 1999; Granot et al. 1999). Meanwhile, we have shown that only a fraction of short GRBs could have $m_{R}<23$ late-time optical afterglows for case 1, while the optical afterglows are readily detected for case 2 . Interestingly, for case 1 we may detect many "dark" bursts, i.e., bursts for which no X-ray, optical, or radio afterglows are observed, when $\Theta$ is relatively large but still within the gamma-ray cone. 
For conical jets the deceleration time should be much shorter than for cylindrical jets, even for a low-density ambient medium, with

$$
t_{\mathrm{dec}}=30\left(\frac{E_{\text {iso }}}{5 \times 10^{51} \mathrm{ergs}}\right)^{1 / 3} n_{-3}^{-1 / 3}\left(\frac{\gamma_{0}}{300}\right)^{-8 / 3} \mathrm{~s} .
$$

The optical afterglow peak time of conical jets is mainly determined by the time when the characteristic frequency $\nu_{m}$ of the afterglow crosses the optical band, which is usually less than $1 \mathrm{hr}$. Therefore, the peak time of the afterglow for a conical jet is wavelength dependent, in contrast with that of a cylindrical jet. Multiwavelength (such as in the $B, V, R$, and $I$ bands) observations of the afterglow are thus useful for discriminating between the cylindrical and conical jets of GRBs. On the other hand, the early afterglow behavior and the late-time asymptotic decay behavior $\left(F_{\nu} \propto t^{-p / 2}-t^{-(p+1) / 2}\right)$ for cylindrical jets are also different from those of conical jets (e.g., Li et al. 2003), although the flux might be too dim to be detected for case 1 when $\Theta$ is large.

In summary, we have studied the afterglow emission of short GRBs, assuming that the outflows are cylindrical in the afterglow phase. We discuss the two cases of whether the jets are still conical (case 1) or have become cylindrical (case 2) in the gamma-ray-emitting phase. Our main results are summarized as follows:

1. In case 1 , since the opening angle of the gamma-rayemitting conical jet is generally larger than the beaming angle of the cylindrical jet during the afterglow phase, observers who see a normal burst of gamma rays are most likely to view an off-axis afterglow. The optical afterglow light curves (for example, the peak time and the flux lever) are very sensitive to the angle between the line of sight and the jet axis and the cross section radius of the cylindrical jets. When the viewing angle $\Theta \lesssim 0.03 \mathrm{rad}$, the optical afterglow at the peak time will have $m_{R}<23$. This implies that a fraction (although not a dominant one) of short GRBs have optical afterglows bright enough for late-time observations by ground-based telescopes. The peak time of the afterglow is generally later than that of the conical jet case, which suggests that continuous follow-up observations up to a few weeks are crucial.

2. For case 2, optical afterglows can be readily detected with $m_{R}<2310 \mathrm{hr}$ after the burst. The light curve, especially the peak time, is also sensitive to the cross section radius.

3. The current observational upper limits of some short GRB afterglows are consistent with the predicted light curves in case 1 with $\Theta>0.02$ rad and the light curves in case 2 with $a \lesssim 10^{15} \mathrm{~cm}$ if typical parameter values are adopted.

The Swift mission, scheduled for launch in the near future, will be sensitive to short GRBs (Gehrels et al. 2004). It will be far more powerful than any previous GRB mission, observing more than 100 bursts $\mathrm{yr}^{-1}$ and performing X-ray and UV/optical afterglow observations in the very early phase. Should Swift discover short GRBs with no X-ray or optical early afterglows, the gamma-ray detector itself will still provide a position accuracy of $1^{\prime}-4^{\prime}$, which is sufficient for optical observations by ground-based telescopes. Therefore, the cylindrical jet model could be tested in the upcoming Swift era.

We are grateful to the referee for his or her valuable comments and suggestions. We would also like to thank Y. F. Huang for his careful reading of the manuscript and helpful comments and Z. G. Dai and T. Lu for useful discussions. This work was supported by the Special Funds for Major State Basic Research Projects, the National 973 Project, the National Natural Science Foundation of China under grants 10403002, 10233010, and 10221001, and an RGC grant ( project HKU 7014/04P) from the Hong Kong government.
Ando, S. 2004, J. Cosmology Astropart. Phys., 6, 7

Belczynski, K., \& Kalogera, V. 2001, ApJ, 550, L183

Beskin, V. S. 1997, Phys.-Uspekhi, 40(7), 659

Bloom, J. S., Kulkarni, S., \& Djorgovski, S. G. 2002, AJ, 123, 1111

Bridle, A. H., \& Perley, R. A. 1984, ARA\&A, 22, 319

Bridle, A. H., Perley, R. A., \& Henriksen, R. N. 1986, AJ, 92, 534

Burrows, C. J., et al. 1996, ApJ, 473, 437

Castro-Tirado, A. J., et al. 2002, A\&A, 393, L55

Cheng, K. S., Huang, Y. F., \& Lu, T. 2001, MNRAS, 325, 599

Dezalay, J. P., Lestrade, J. P., Barat, C., Talon, R., Sunyaev, R., Terekhov, O., \& Kuznetsov, A. 1996, ApJ, 471, L27

Fan, Y. Z., Zhang, B., Kobayashi, S., \& Mészáros, P. 2004, ApJL, submitted (astro-ph/0410060)

Fendt, C., \& Ouyed, R. 2004, ApJ, 608, 378

Fenimore, E. E., et al. 2004, GCN Circ. 2735, http://gen.gsfc.nasa.gov/gcn/ gen $3 / 2735 . g c n 3$

Fox, D. B., \& Moon, D. S. 2004, GCN Circ. 2734, http://gcn.gsfc.nasa.gov/ $\mathrm{gcn} / \mathrm{gcn} 3 / 2734 . \mathrm{gcn} 3$

Frail, D. A., et al. 2001, ApJ, 562, L55

Gehrels, N., et al. 2004, ApJ, 611, 1005

Golenetskii, S., Aptekar, R., Mazets, E., Pal'Shin, V., Frederiks, D., \& Cline, T. 2004, GCN Circ. 2754, http://gcn.gsfc.nasa.gov/gcn/gcn3/2754.gcn3

Gorosabel, J., et al. 2002, A\&A, 383, 112

Granot, J., Panaitescu, A., Kumar, P., \& Woosley, S. E. 2002, ApJ, 570, L61

Granot, J., Piran, T., \& Sari, R. 1999, ApJ, 527, 236

Hjorth, J., et al. 2003, Nature, 423, 847

Huang, Y. F., Dai, Z. G., \& Lu, T. 1999, MNRAS, 309, 513

Huang, Y. F., Gou, L. J., Dai, Z. G., \& Lu, T. 2000, ApJ, 543, 90

Huang, Y.-F., Tan, C. Y., Dai, Z. G., \& Lu, T. 2002, Chinese Astron. Astrophys., 26, 414
REFERENCES

Hurley, K., et al. 2002, ApJ, 567, 447

Kalogera, V., et al. 2004, ApJ, 601, L179

Kehoe, P., et al. 2001, ApJ, 554, L159

Klotz, A., Boër, M., \& Atteia, J. L. 2003, A\&A, 404, 815

Kouveliotou, C., Meegan, C. A., Fishman, G. J., Bhat, N. P., Briggs, M. S.,

Koshut, T. M., Paciesas, W. S., \& Pendleton, G. N. 1993, ApJ, 413, L101 Kumar, P., \& Granot, J. 2003, ApJ, 591, 1075

Li, W., Filippenko, R., Chornock, R., \& Jha, S. 2004, GCN Circ. 2748, http:// gcn.gsfc.nasa.gov/gcn/gcn3/2748.gcn3

Li, Z., Dai, Z. G., \& Lu, T. 2003, MNRAS, 345, 1236

Lobanov, A. P., Gurvits, L. I., Frey, S., Schilizzi, R. T., Kawaguchi, N., \& Pauliny-Toth, I. I. K. 2001, ApJ, 547, 714

MacFadyen, A. \& Woosley, S. E. 1999, ApJ, 524, 262

Mazets, E., et al. 1981, Ap\&SS, 80, 3

Mészáros, P., \& Rees, M. J. 1997, ApJ, 476, 232

Paczyński, B. 1998, ApJ, 494, L45

Panaitescu, A., \& Kumar, P. 2001, ApJ, 560, L49

2002, ApJ, 571, 779

Panaitescu, A., Kumar, P., \& Narayan, R. 2001, ApJ, 561, L171

Perna, R., \& Belczynski, K. 2002, ApJ, 570, 252

Ray, T. P., Mundt, R., Dyson, J. E., Falle, S. A. E. G., \& Raga, A. C. 1996, ApJ, 468, L103

Rossi, E., Lazzati, D., \& Rees, M. J. 2002, MNRAS, 332, 945

Rosswog, S., Ramirez-Ruiz, E., \& Davies, M. B. 2003, MNRAS, 345, 1077

Ruffert, M., \& Janka, H. T. 1999, A\&A, 344, 573

Sari, R., Piran, T., \& Narayan, R. 1998, ApJ, 497, L17

Stanek, K. Z., et al. 2003, ApJ, 591, L17

van Paradijs, J., et al. 1997, Nature, 386, 686

Vietri, M. 1997, ApJ, 478, L9 
Vlahakis, N., \& Königl, A. 2003a, ApJ, 596, 1080 2003b, ApJ, 596, 1104

Wang, X. Y., Dai, Z. G., \& Lu, T. 2000, MNRAS, 319, 1159

Waxman, E. 1997a, ApJ, 485, L5

1997b, ApJ, 489, L33

Wijers, R. A. M. J., \& Galama, T. J. 1999, ApJ, 523, 177
Wijers, R. A. M. J., Rees, M. J., \& Mészáros, P. 1997, MNRAS, 288, L51 Woosley, S. E. 1993, ApJ, 405, 273

Zhang, B., Dai, X., Lloyd-Ronning, N. M., \& Mészáros, P. 2004, ApJ, 601, L119

Zhang, B., \& Mészáros, P. 2002, ApJ, 571, 876 